\title{
Experimental Analysis of the Distribution of Traction Coefficient in the Shoe-Ground Contact Area during Running
}

\author{
Kenta Moriyasu $^{1,2)^{*}}$, Tsuyoshi Nishiwaki ${ }^{1)}$, Takeshi Yamaguchi ${ }^{2)}$ and Kazuo Hokkirigawa ${ }^{2)}$ \\ ${ }^{1)}$ Institute of Sport Science, ASICS Corporation \\ 6-2-1, Takatsukadai, Nishi-ku, Kobe, Hyogo 651-2271 Japan \\ ${ }^{2)}$ Graduate School of Engineering, Tohoku University \\ 6-6-01 Aramaki-Aza-Aoba, Aoba-ku, Sendai, Miyagi 980-8579, Japan \\ *Corresponding author: moriyasu-ke@asics.co.jp
}

( Manuscript received 23 September 2012; accepted 31 October 2012; published 31 December 2012 )

\begin{abstract}
Relationship between shoe grip properties and distributions of traction coefficient, which is obtained from horizontal ground reaction force (GRF) divided by normal GRF, were experimentally investigated during running. The experiments were conducted with sensor shoes mounted miniature triaxial force sensors for the measurement of GRF distributions in contact area. In order to clarify influence of the grip property on GRF vectors distributions and traction coefficient distributions, two typed sensor shoes having different outer sole materials with high/low friction coefficients were developed. The results showed that traction coefficients for the low grip typed shoe decreased in the whole contact area at the end of stance phase during running. Furthermore, it was confirmed that contact area, directions of GRF vectors and traction coefficients locally changed depending on the grip property. As a result of relationship between distributions of propulsion force components at 19 local positions and stride length, production of propulsion force beneath toe area can efficiently acquire sufficient stride length to keep running speed.
\end{abstract}

Keywords: shoes, grip property, ground reaction force, traction coefficient distribution, tribology

\section{Introduction}

Recently, the popularity of running has been growing with becoming more health conscious. Running shoes are designed depending on runners' skills and they play important roles to prevent injury and enhance performance. For example, cushioning, stability and flexibility are required for recreational runners, while lightness and air permeability are important for elite runners. Within these various functions, grip property of the shoes is of crucial importance regardless of the usage conditions. Especially, it is predicted that low grip increases the risk of falling accidents caused by induced slips and decreases running performance.

Grip condition between shoe sole and ground surface is generally figured out with horizontal ground reaction force (GRF) and traction coefficient, which is calculated by horizontal GRF divided by normal GRF. In running and walking, slips will not occur theoretically as long as traction coefficient at the shoe-ground contact interface is smaller than the friction coefficient. Previous studies have discussed these, which cause falls due to induced slips during walking [1-3]. These studies have demonstrated that center of mass position and hip movement in vertical direction relate to the slip onset during walking on slippery surfaces. In terms of performance in running, it has been said that stride length, propulsion force and propulsion impulse increase with the running velocity [4]. However, it is difficult to understand positions where high grip propety is required in the design process of the shoe, because GRFs measured with force plates in these studies are corresponding to the resultant forces in the whole contact area between shoe and ground surface. Namely, it is necessary to detect positions, in the contact area, where horizontal force or the traction coefficient takes high/low value. According to previous reports, shoes mounted force sensors, accelerometers and goniometers have been developed [5]. However, the practical motion was obstructed by shoe weight gain and sensors loaded on the shoe upper. In our previous study [6], a novel sensor shoe mounted miniature triaxial force sensors was developed to measure GRFs and traction coefficient distributions in the shoe-ground contact area. It was demonstrated distributions of GRFs and traction coefficients are obtained during running using the sensor shoe, and it 
would be expected that considering the relationship between these distributions indicates appropriate areas in the shoe outer sole where high grip property is necessary.

The purpose of this study was to investigate relationship between shoe grip properties and distributions of traction coefficient in the shoe-ground contact area during running. Two typed sensor shoes with different outer sole materials having high/low friction coefficients were developed. By using the shoes, influence of the grip property on GRF vector distributions and traction coefficient distributions were clarified. Furthermore, relationship between distributions of propulsion force components in the shoe-ground contact area and stride length was also discussed.

\section{Experimental Methods}

Four healthy male subjects [age, $31.0( \pm 8.3)$ years; height, $1.76( \pm 0.04) \mathrm{m}$; weight, $67.6( \pm 5.57) \mathrm{kg}]$ participated in this study. The experiment was carried out on polyurethane coated flooring. A running speed was set to $4.17 \mathrm{~m} / \mathrm{s}$. A motion capture system (ViconT; Vicon Motion Systems Ltd., Oxford, UK) and a force plate (Model 9287A; Kistler Instrument Corp, Winterthur, Switzerland) were used with the sensor shoe.

Fig. 1 shows the sensor shoe [6] with 6 triaxial force sensors and 13 dummies at the bottom of the sole. These

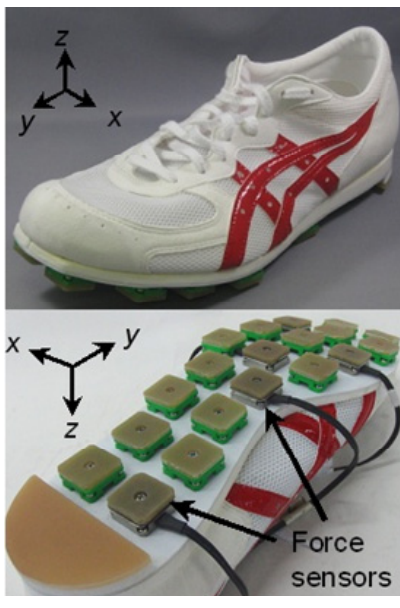

(a) Sensor shoe

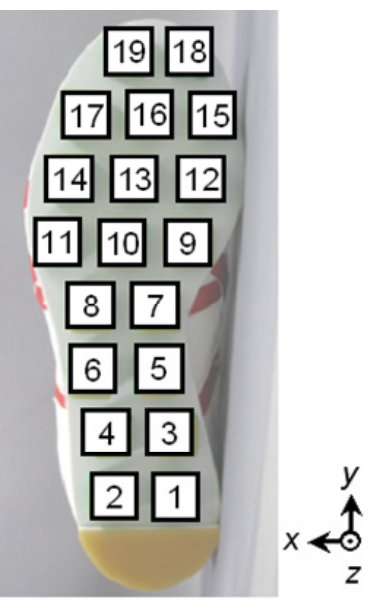

(b) Sensor position
Fig. 1 Sensor shoe mounted tri-axial force sensor sensors whose rate capacities are $250 \mathrm{~N}, 250 \mathrm{~N}$, and 500 $\mathrm{N}$ in $x, y$, and $z$ direction, respectively, were used to measure GRFs distributions in the shoe-ground contact are. Force components can be measured at 19 positions by changing the sensor position. Force components obtained from the sensors were defined as $f x, f y$, and $f z$. Subscripts $x, y$, and $z$ are corresponding to lateral, longitudinal and normal direction of the shoe, respectively. Total shoe weight with 6 sensors and 13 dummy devices is $272.5 \mathrm{~g}$, which is less than a commercial running shoe weight. Fig. 2 is section views of sensor devices showing sensor devices' compositions selected. These devices consist of the force sensor,

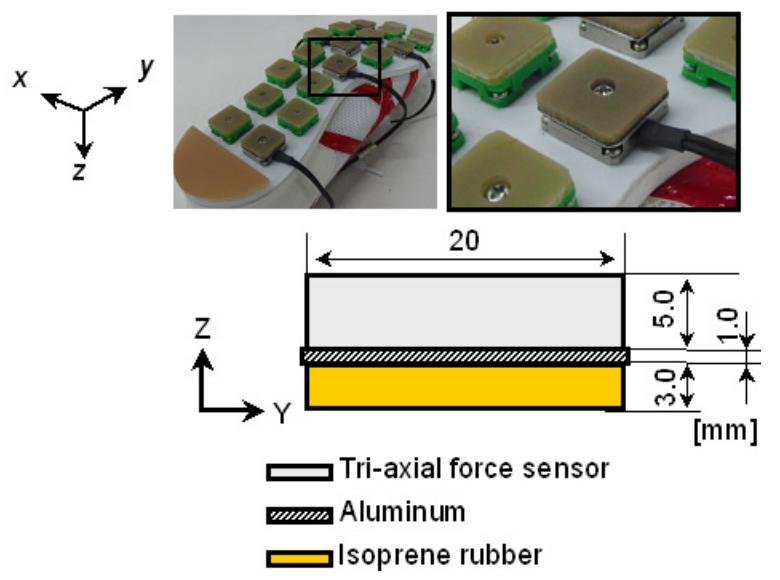

(a) Type A

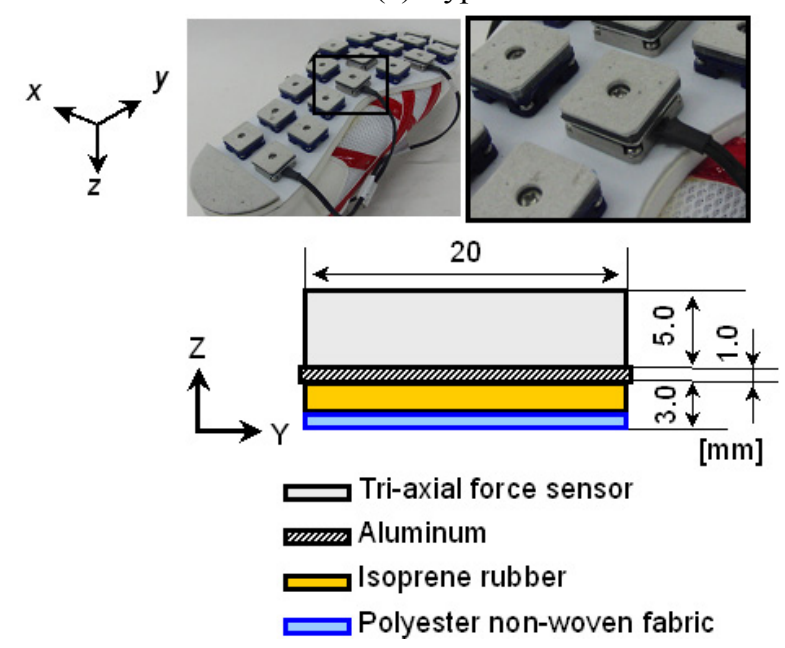

(a) Type A

Fig. 2 Section views of sensor devices

Table 1 comparison of friction coefficient between outer sole material and floor surface (JIS K7312)

\begin{tabular}{|c|c|c|}
\hline & Static friction coefficient $\mu_{s}$ & Dynamic friction coefficient $\mu_{d}$ \\
\hline $\begin{array}{c}\text { Isoprene rubber } \\
\text { (TypeA) }\end{array}$ & $1.528 \pm 0.137$ & $1.539 \pm 0.133$ \\
\hline $\begin{array}{c}\text { Polyester non-woven fabric } \\
\text { (TypeB) }\end{array}$ & $0.248 \pm 0.004$ & $0.222 \pm 0.004$ \\
\hline
\end{tabular}


aluminum and outer sole materials. In order to modify the grip property of Type A and B, different typed outer sole materials were applied. Here, isoprene rubber was applied to that of Type $\mathrm{A}$, and isoprene rubber and polyester non-woven fabric were applied to the outer sole of Type B. Static and dynamic friction coefficients, $\mu_{s}$ and $\mu_{d}$ were listed in Table 1 . As shown in Table 1 , both $\mu_{s}$ and $\mu_{d}$ in isoprene rubber are approximately five times higher than those in polyester non-woven fabric.

To measure GRF distribution, sensor arrangement was changed as shown in Table 2. 4 types of the sensor arrangements were selected to measure GRF components at 19 local positions. 6-8 trials were conducted in each arrangement. In order to eliminate influences of contact condition between the shoe and the ground, all outputs from the force sensors were transformed from the sensors' local coordinates to global coordinate by an affine transformation. The local coordinates at the position $i$ was defined based on 3-4 reflective markers' coordinates obtained from the motion capture system as shown in Fig. 3. $x$ axis of the local coordinates was aligned with the vectors directions represented with $\mathrm{M}_{i}$ and $\mathrm{L}_{i}$ coordinates. Subscript $i, i=$ 1-9 denotes the position numbers in Fig. 3.

In data acquisition, 2 analog to digital converters, AD1 (DSS300-01; TecGihan Co. Ltd. Japan), AD2 (IRC-003; Inter Reha Co. Ltd. Japan) were used. All outputs from the force sensors and the conventional force plate were collected by AD1 and AD2. Sampling rates were $1.0 \mathrm{kHz}$ in both the sensor shoe and the force plate. Force data during stance phase was extracted based on GRF obtained from the force plate. Average data, $f x_{i}, f y_{i}, f z_{i}$ and resultant GRFs, $F x, F y$ and $F z$ were

Table 2 Sensor arrangements

\begin{tabular}{|c|c|}
\hline & Position numbers of sensors \\
\hline Type1 & $i=1,2,4,17,18,19$ \\
\hline Type 2 & $i=2,3,4,15,16,17$ \\
\hline Type3 & $i=2,5,6,12,13,14$ \\
\hline Type4 & $i=2,7,8,9,10,11$ \\
\hline
\end{tabular}

calculated from the all outputs obtained from four subjects. Subscript $i, i=1-19$ corresponds to the position numbers in Fig. 1.Traction coefficients, $\Phi$ in whole contact area and $\phi_{i}(i=1-19)$ at 19 local positions were calculated based on the resultant GRFs (measured with the conventional force plate) and the force components (measured with the force sensor), respectively. $\Phi$ and $\phi_{i}$ are represented by the following Equations (1) and (2),

$$
\begin{aligned}
& \Phi=\left|\frac{\sqrt{F x^{2}+F y^{2}}}{F z}\right| \\
& \phi_{i}=\left|\frac{\sqrt{f x_{i}^{2}+f y_{i}^{2}}}{f z_{i}}\right|, i=1-19
\end{aligned}
$$

To investigate influences of maximal propulsion forces, $F y^{\max }$ and $f y_{i}^{\max }$ and propulsion impulses on running performance, $P I$ and $p i_{i}$ are computed. $F y^{\max }$ and $f y_{i}{ }^{\max }$ are corresponding to the maximal $F y$ and $f y_{i}$, respectively. $P I$ and $p i_{i}$ are calculated by the following Equations (3) and (4),

$$
\begin{aligned}
P I & =\frac{\sum F y^{+}}{B M} \\
p i_{i} & =\frac{\sum f y_{i}^{+}}{B M}, i=1-19
\end{aligned}
$$

where, $F y^{+}$and $f y_{i}^{+}$, are positive $F y$ and $f y_{i}$ defined as propulsion force components, $B M$ is the subject body mass.

\section{Results}

Fig. 4 shows time histories of traction coefficient, $\Phi$ in the contact area during stance phase in running. $\Phi$ has a peak at $20 \%$ and increases from $60 \%$ to $90 \%$ of stance phase. Compared Type A with Type B, $\Phi$ in Type B did not increase after $70 \%$ of stance phase, because $\Phi$ possibly reached the static friction coefficient $\left(\mu_{s}=\right.$ $0.248 \pm 0.004)$ of polyester non-woven fabric as shown in Table 1. Significant differences were observed between $\Phi$ in Type A and $\Phi$ in Type B at $20 \%$ of stance phase and from $60 \%$ to $90 \%$ of stance phase. As

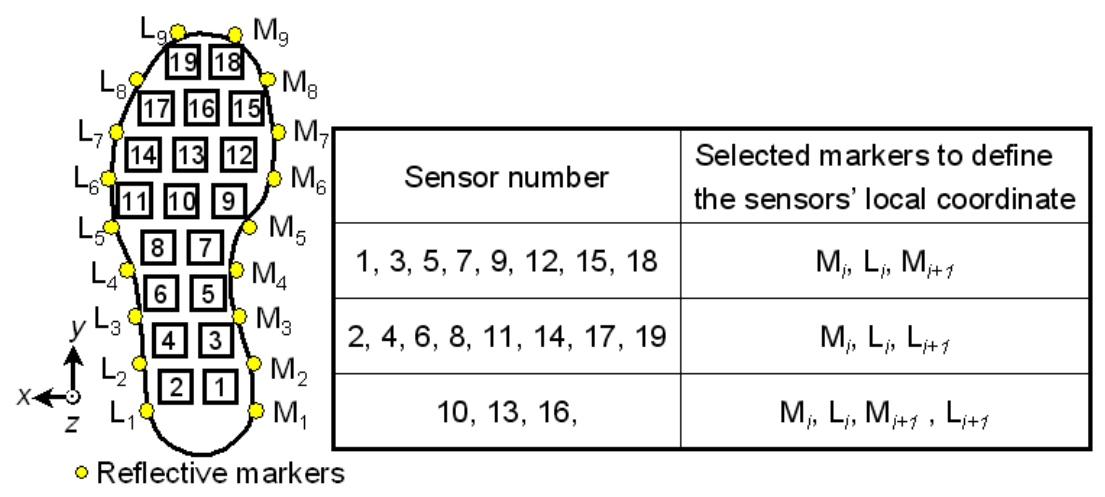

Fig. 3 Definition of the sensors' local coordinates 
mentioned above, slip onset is avoided when traction coefficient at the shoe-ground contact interface is smaller than the friction coefficient in running. Namely, these results well explain that slip occurs at the end of stance phase with Type B and slip onset is not likely at the beginning of stance phase. Average value of the backward slip distance for Type B calculated form y coordinate of $\mathrm{M}_{2}$ marker was $33.3 \pm 16.0 \mathrm{~mm}$.

$\phi_{i}$ histories during stance phase in running are shown in Fig. 5. Closed circle means that traction coefficient for Type A and Type B at each time of stance phase was significantly different $(p<0.05)$. With increasing $i$, changing the position from heel to toe, both the start and end times of the contact period while the forces acting on the sensor delayed. $\phi_{1}$ and $\phi_{2}$ positioned in rearfoot area initially increased during stance phase. Peaks of $\phi_{18}$

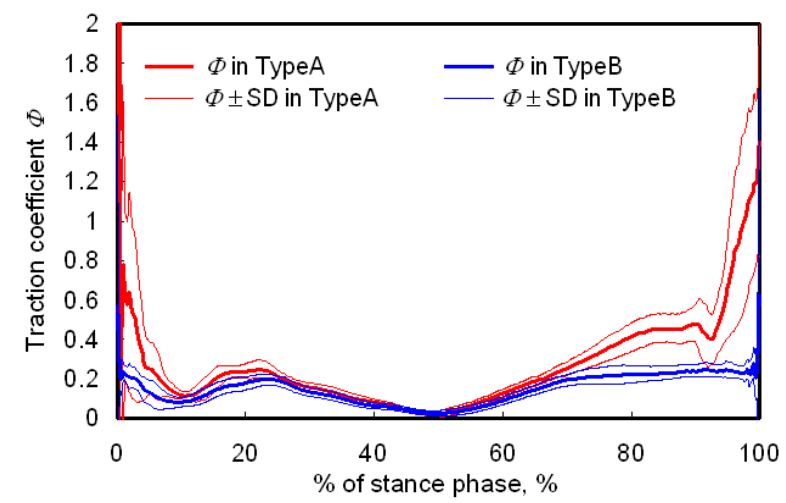

Fig. 4 Time histories of traction coefficient $\Phi$ and $\phi_{19}$ appeared at the end of stance phase. It can be confirmed that some prominent peaks appear at the start and end times of the contact period while the forces acting on the sensor. There is a possibility that frontal/trailing edge of the sensor only contacts on the ground at these phases due to inclination of the sensor. Horizontal force component might contain normal force component under the influence of these edge contacts and be increased. Namely, calculated $\phi_{i}$ relatively get increased. As described in experimental method, in order to eliminate influences of contact condition between the shoe and the ground, all outputs from the force sensors were transformed from the sensors' local coordinates to global coordinate by an affine transformation. However, the prominent peaks possibly include the influence of the sensor's inclination.

Compared Type A with Type B, contact period became longer at $i=1-17$ with Type $\mathrm{B}$ and $\phi_{i}$ in Type $\mathrm{B}$ became lower than those in Type A because of slip. $\phi_{18}$ and $\phi_{19}$ in Type A were higher than those in Type B during the entire contact period. Peak values of $\phi_{i}$ at $i=$ $1-5,8,9,12,13,15,18,19$ in Type B were significantly lower than those of Type A. In addition, these values are equal to or higher than the static/dynamic friction coefficient of the outer-sole. Namely, slips occur in not only the whole contact area but also local contact area for Type B. These results clearly demonstrated that traction coefficient distributions in the shoe-ground contact area measured by using the sensor shoes provide information of where in the contact area and when slips initiate.

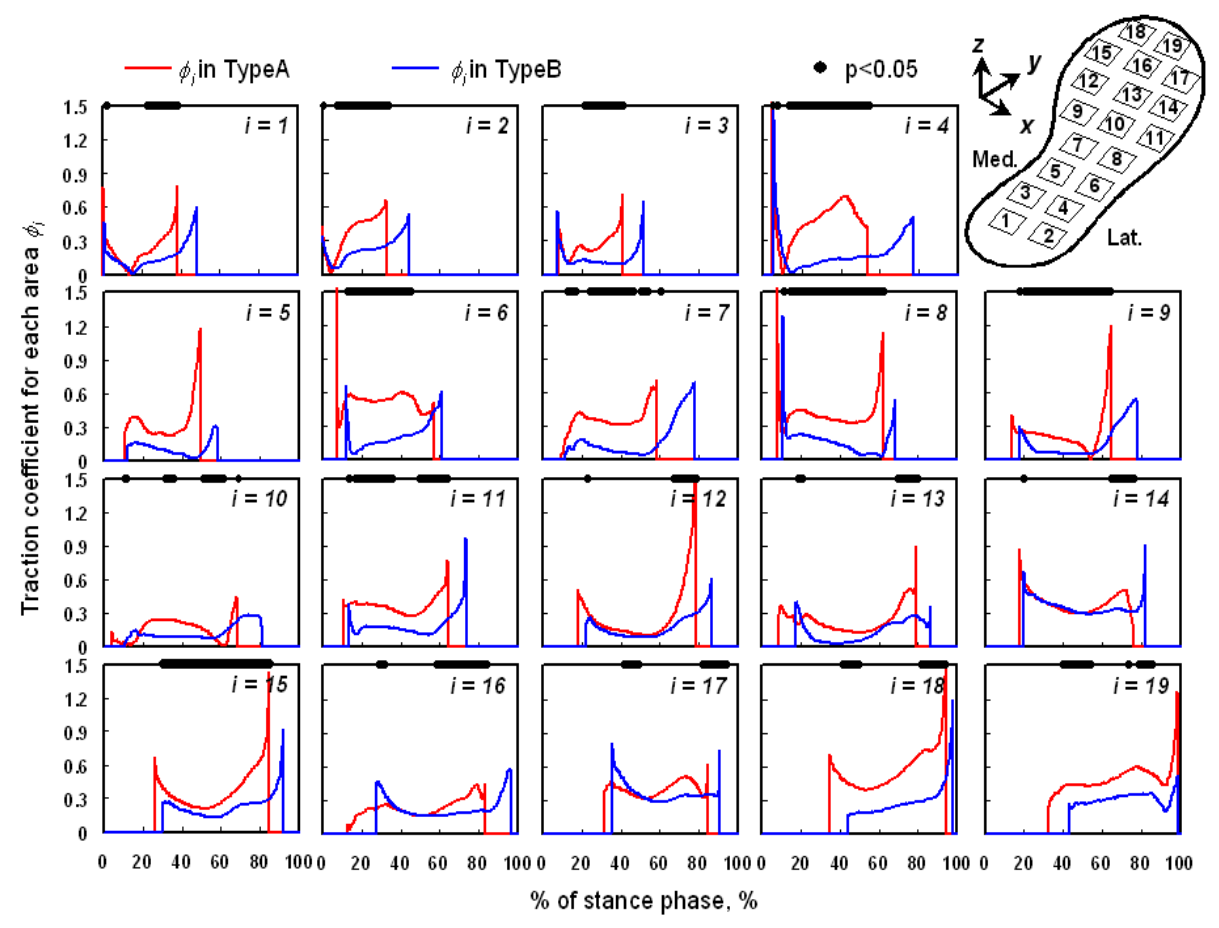

Fig. 5 Time histories of traction coefficient $\phi_{i}$ 


\section{Discussion}

In order to clarify crucial positions for grip, GRF vectors and $\phi_{i}$ distributions were compared. Distributions of $f z_{i}$ vectors, horizontal force, $f h_{i}$ vectors and $\phi_{i}$ at $20 \%, 50 \%$ and $80 \%$ of stance phase are shown in Fig. 6. $f h_{i}$ denotes the resultant force of $f x_{i}$ and $f y_{i}$. It was confirmed that $f h_{i}, f z_{i}$ and $\phi_{i}$ had different distributions depending on the stance phase. $f h_{i}$ and $\phi_{i}$

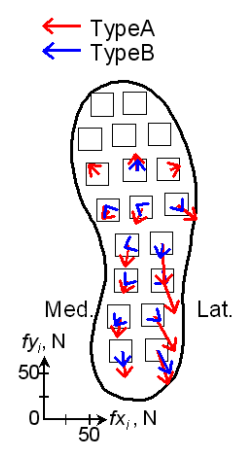

$f h_{i}$ vectors

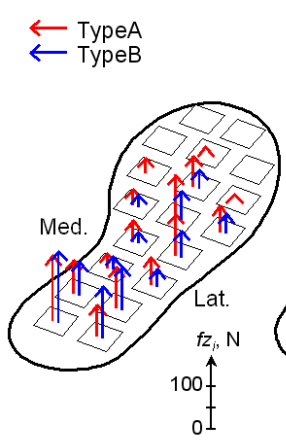

$f z_{i}$ vectors
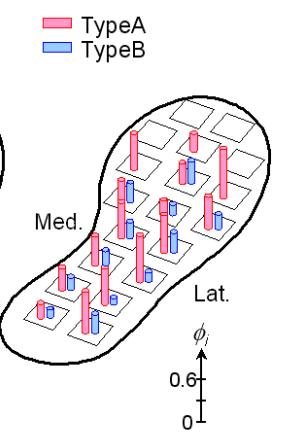

$\phi_{i}$ (a) $20 \%$ of stance phase

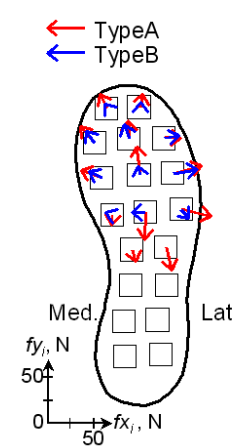

fh, vectors

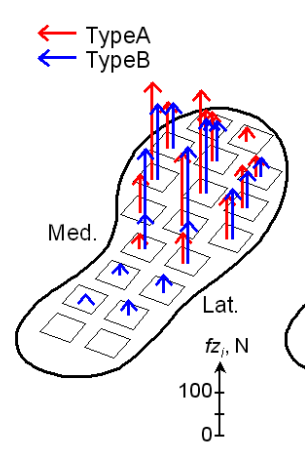

$f z_{i}$ vectors
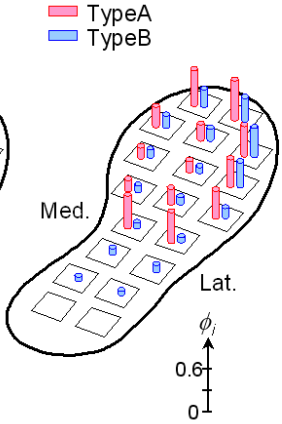

$\phi_{i}$ (b) $50 \%$ of stance phase

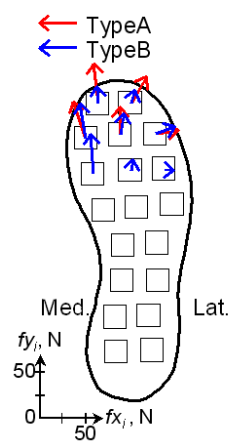

$f h_{i}$ vectors

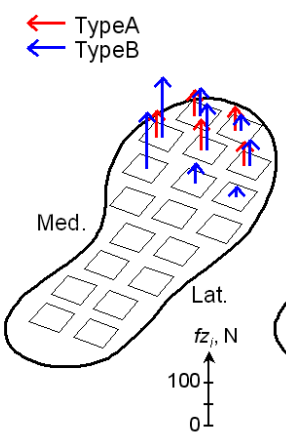

$z_{i}$ vectors

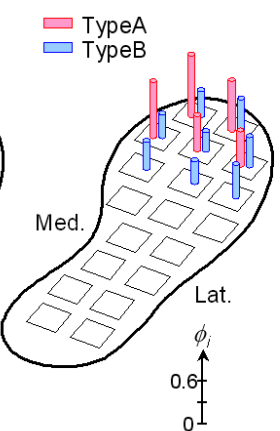

$\phi_{i}$ (c) $80 \%$ of stance phase

Fig. 6 Distributions of $f h_{i}$ vectors, $f z_{i}$ vectors and $\phi_{i}$ at $20 \%, 50 \%$ and $80 \%$ of stance phase distributions were particularly different between Type A and Type B at $20 \%$ and $80 \%$ of stance phase. For example, $\phi_{i}$ at $i=2,4,6,8$ and 11 with Type A were higher than those with Type B at $20 \%$ of stance phase. In the same way, it can be confirmed that $\phi_{i}$ at $i=18$ and 19 with Type A were higher than those with Type B at $80 \%$ of stance phase. Fig. 7 shows schematic view of slip and no-slip area at $20 \%, 50 \%$, and $80 \%$ of stance phase. Red-hatched area denotes no-slip area where $\phi_{i}$ is lower than $\mu_{\mathrm{s}}$. Blue-hatched area denotes slip area where $\phi_{i}$ reaches $\mu_{\mathrm{s}}$. As shown in Figs. 6 and 7, contact area was enlarged at 50 and $80 \%$ of stance phase in the case of Type B. Slip initiated in forefoot lateral side at $50 \%$ of stance phase and the slip area was enlarged from 50 to $80 \%$ of stance phase with Type B. Furthermore, it can be confirmed that the slip area was over $75 \%$ of whole contact area at $80 \%$ of stance phase. On the other hand, slip did not occur in whole contact area with Type A. In comparison of $f h_{i}$ vectors, it was confirmed that $f h_{i}$ vectors were affected by slip occurrence; $f h_{i}$ vectors in TypeB dominantly acted in forward direction compared with those in Type A at $80 \%$ of stance phase as shown in Fig. 6(c). These results explain that not only forward/backward direction but also mediolataral direction must be considered in the grip design process of running shoe.

It has been said that stride length, propulsion force and propulsion impulse increase with running velocity [4]. Weyand [4] indicated that faster top running speeds were achieved with greater GRF. In constant speed running, it was clarified that slip occurs at the end of stance phase with low grip typed shoe. Slips disturb production of horizontal force for braking and propulsion, because traction coefficient doesn't get higher than friction coefficient. Namely, slip in not only whole contact area but also local contact area will affect stride length and propulsion force in constant speed running. Therefore, relationships between stride length and propulsion force produced at each sensor position for Type A and Type B were compared. For the comparisons, maximal propulsion forces, $f y_{i}^{\max }$ obtained from each sensor position and the propulsion impulse, $p i_{i}$ were calculated. Stride length were calculated from a distance between right heel contact point and left heel contact point based on the marker coordinates attached on heel of the shoes.

Table 3 shows correlation coefficients between stride length and $f y_{i}^{\max } / p i_{i}$. In comparison of correlation coefficients between stride length and $f y_{i}^{\max }$, there are significant correlations at $i=9,10,15,18$ and 19. In addition, there are significant correlations between stride length and $p i_{i}$ at $i=15,18$ and 19. Interestingly, negative correlation was observed at $i=9$ and 10 while $i$ $=15,18$ and 19 have positive correlation. In the case of Type B, contact area was enlarged during second half of stance phase and propulsion force was produced at not only $i=15,18$ and 19 but also $i=9$ and 10. Judging from these results, production of propulsion force at $i=$ 


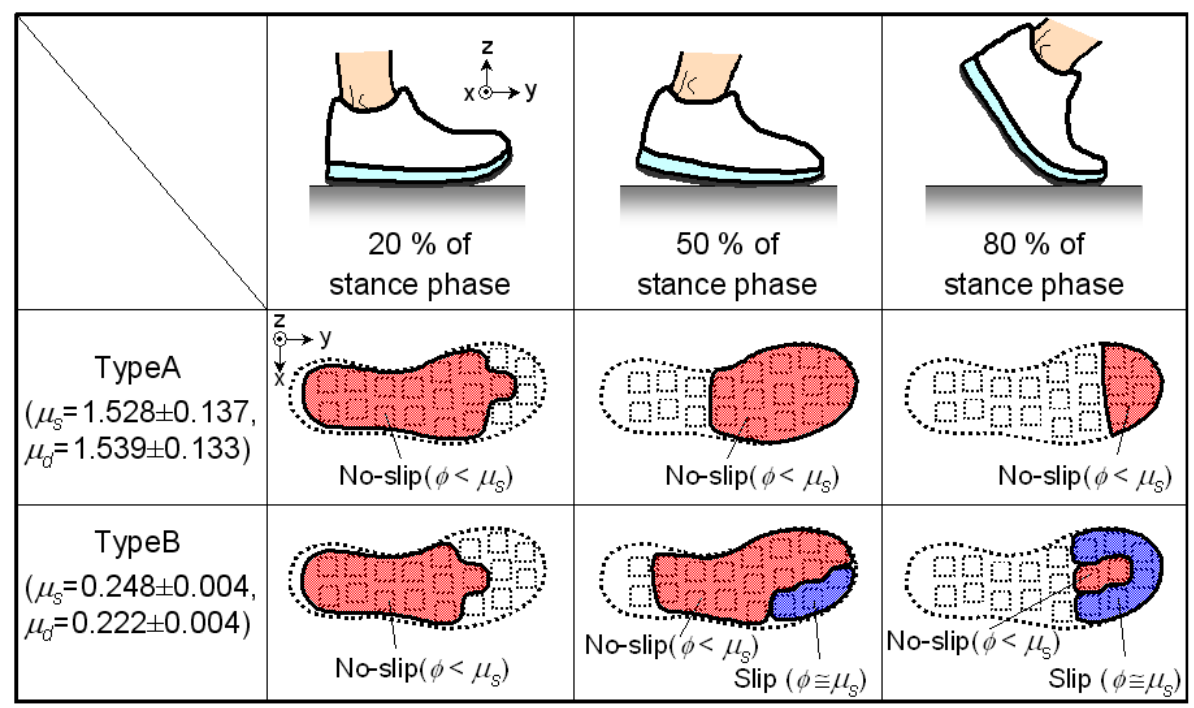

Fig. 7 Schematic view of slip and no-slip area at $20 \%, 50 \%$ and $80 \%$ of stance phase during running

Table 3 Correlation coefficients between $f y_{i}^{\max } / p i_{i}$ and stride length

\begin{tabular}{|c|c|c|}
\hline & \multicolumn{2}{|c|}{ Correlation coefficients $\left.*^{*} p<0.05\right)$} \\
\hline Sensor number & Stride length vs $f y_{i}^{\max }$ & Stride length vs $p i_{i}$ \\
\hline$i=9$ & $0.1947^{*}$ & 0.1376 \\
\hline$i=10$ & $0.2109^{*}$ & 0.0214 \\
\hline$i=12$ & 0.0008 & 0.0112 \\
\hline$i=13$ & 0.0144 & 0.0003 \\
\hline$i=14$ & 0.0581 & 0.1146 \\
\hline$i=15$ & $0.7210^{*}$ & 0.4461 \\
\hline$i=16$ & 0.0017 & 0.0324 \\
\hline$i=17$ & 0.0582 & 0.0005 \\
\hline$i=18$ & $0.5213^{*}$ & $0.5449 *$ \\
\hline$i=19$ & $0.3047^{*}$ & $0.2483 *$ \\
\hline & Stride length vs $F y^{\max }$ & Stride length vs $P I$ \\
\hline Resultant force & 0.1574 & 0.0521 \\
\hline
\end{tabular}

15, 18 and 19 beneath toe area can efficiently acquire sufficient stride length to keep running speed. Under low grip condition, running speed is sustained by the production of $f y_{i}^{\max }$ at $i=9$ and 10 beneath the ball.

\section{Conclusions}

In this research, two typed sensor shoes with different outer sole materials having high/low friction coefficients were developed and relationship between shoe grip properties and distributions of traction coefficient in the shoe-gournd contact area between shoe sole and ground surface during running was clarified. It was confirmed that $\phi$ with low grip typed shoe decreases in whole contact area at the end of stance phase during running. Depending on the grip property, directions of GRF vectors and $\phi$ locally changed. Furthermore, production of propulsion force beneath toe area can efficiently acquire sufficient stride length to keep running speed based on relationship between propulsion force distributions and stride length.

\section{References}

[1] Yamaguchi, T. and Hokkirigawa, K., “"Walking-Mode Maps' Based on Slip/Non Slip Criteria," Industrial Health, 46, 1, 2008, 23-31.

[2] Yamaguchi, T., Hatanaka, S. and Hokkirgawa, K., "Effect of Step Length and Walking Speed on Traction Coefficient and Slip between Shoe Sole and Walkway," Tribology Online, 3, 2, 2008, 59-64.

[3] Yang, F., Bhatt, T. and Pai, Y. C., "Role of Stability and Limb Support in Recovery Against a Fall Following a Novel Slip Induced in Different Daily Activities," Journal of Biomechanics, 42, 12, 
2009, 1903-1908.

[4] Weyand, P. G., Sternlight, D. B., Bellizzi, M. J. and Wright, S., "Faster Top Running Speeds are Achieved with Greater Ground Forces not more Rapid Leg Movements," Journal of Applied Physiology, 89, 5, 2000, 1991-1999.

[5] Bamberg, S. J. M., Benbasat, A. Y., Scarborough, D. M., Krebs, D. E. and Paradiso, J. A., "Gait
Analysis Using a Shoe-Integrated Wireless Sensor System," IEEE Transactions on Information Technology in Biomedicine, 12, 4, 2008, 413-423.

[6] Moriyasu, K., Nishiwaki, T., Yamaguchi, T. and Hokkirigawa, K., "New Technique of Three Directional Ground Reaction Force Distributions," Footwear Science, 2, 2, 2010, 57-64. 